\title{
The Stress Rate of Hyperelasticity Based on the Updated Lagrangian Formulation*
}

\author{
Akihiro MATSUDA**, Osamu WATANABE*** and René DE BORST ${ }^{* * * *}$
}

\begin{abstract}
A rate-type constitutive equation of compressible isotropic hyperelasticity and a finite element formulation based on the Updated Lagrangian approach are proposed. The constitutive equations of compressible hyperelasticity are simplified by introducing modified tensors in an intermediate configuration, which separates the volumetric deformation from the distortional deformation. Selective integration and a mixed pressure/displacement formulation have been adopted to avoid volumetric locking and the hourglass modes. The deformation of a simple rectangle block was simulated to show the effect of the choice of the finite elements and the integration methods on the stability and the accuracy of proposed method. Moreover, loading tests were carried out on a prototype specimen of a vibration control device made from circumferential rubber parts and reinforcing steel layers in order to evaluate applicability of the proposed formulation to such devices.
\end{abstract}

Key Words: Finite Element Method, Hyperelasticity, Updated Lagrangian Formulation.

\section{Introduction}

The Lagrangian formulation is well established and widely used for the analysis of solid and structures ${ }^{(1)}$. In a Lagrangian formulation, stress and strain are referred to a known configuration. Commonly, either the undeformed configuration is chosen as this reference state, the so-called Total Lagrangian formulation, or the most recently calculated configuration, the so-called Updated Lagrangian configuration ${ }^{(2),(3)}$. The choice for one formulation or the other depends on the constitutive equation and the numerical efficiency.

Hyperelasticity has commonly been used for numerical modeling of rubberlike materials. Rubberlike materials are almost incompressible, which means that the bulk modulus is much larger than the shear modulus. As a consequence, traditional displacement-based low-order finite elements exhibit volumetric locking or hourglass modes, depending on the employed integration rule ${ }^{(4)}$. A mixed formulation with the nodal mean stress as unknown

* Received 16th June, 2003 (No. 01-0820)

** Central Research Institute of Electric Power Industry, 1646 Abiko, Abiko, Chiba 270-1194, Japan

*** Institute of Engineering Mechanics and Systems, University of Tsukuba, Tsukuba, Ibaraki 305-8573, Japan

**** Faculty of Aerospace Engineering, Delft University of Technology, Kluyverweg 1 Delft, 2600 GB Delft, The Netherlands was proposed for the analysis of (nearly incompressible) elasto-plastic problems ${ }^{(5)}$.

In hyperelastic formulations, the constitutive equation is naturally derived in the terms of the Second PiolaKirchhoff stress ${ }^{(6)}$. In such a case, the Total Lagrangian formulation can be used efficiently and the performance of displacement/pressure mixed methods and selective integration algorithm has been addressed in the literature ${ }^{(7)}$. However, there is a tendency to use rubberlike materials in composite structures together with elasto-plastic materials ${ }^{(8)}$. Then, an Updated Lagrangian formulation may be more appropriate, thus necessating rewriting the hyperelastic formulations in terms of stress rates.

Moreover, the pressure stress of hyperelasticity has initial value. Following two equations were proposed to ignore the initial stress of hyperelasticity ${ }^{(9)}$. The first potential is

$$
\begin{aligned}
\bar{W} & =W\left(I_{1}, I_{2}\right) \\
& -\left\{\left.\frac{\partial W}{\partial I_{1}}\right|_{I_{1}=3, I_{2}=3}+\left.2 \frac{\partial W}{\partial I_{2}}\right|_{I_{1}=3, I_{2}=3}\right\} \cdot g\left(I_{3}\right),
\end{aligned}
$$

where $I_{1}$ and $I_{2}$ are the first and second invariants of the right Cauchy-Green deformation tensor, and $g$ is a function of third invariant $I_{3}$. The next equation is

$$
\bar{W}=W(\bar{C})=W\left(\bar{I}_{1}, \bar{I}_{2}\right),
$$

where $\bar{I}_{1}$ and $\bar{I}_{2}$ are the first and second invariants preserving volume change. 
In the previous report, we use Eq. (1) for potential energy to ignore the initial stress of hyperelasticity ${ }^{(10)}$. Above equations show the similar stress-strain relation in small strain. However the deviatoric stress part given from Eq. (1) include mean components in finite strain.

In this contribution, the stress rate of isotropic hyperelasticity, either be it incompressible or compressible, is derived in terms of the Truesdell stress rate using by Eq. (2). Subsequently, the governing finite element equations are derived by utilizing a standard mixed displacement/pressure approach and selective integration. The effect of the integration rule and the time stepping algorithm are evaluated by comparison with results obtained from a Total Lagrangian formulation. Finally, loading tests on rubber and steel composite bearings are simulated to assess the applicability of proposed formulation to such structures.

\section{Theoretical Developments}

The initial and current configurations are denoted as $C_{0}$ and $C$, respectively. When the material particle initially located at some position $\boldsymbol{X}$ moves to a new position $\boldsymbol{x}$, the deformation gradient tensor $\boldsymbol{F}$ is given by

$$
\boldsymbol{F}=\frac{\partial \boldsymbol{x}}{\partial \boldsymbol{X}}
$$

The deformation gradient tensor $\boldsymbol{F}$ is decomposed as:

$$
\boldsymbol{F}=\overline{\boldsymbol{F}} \cdot J^{\frac{1}{3}} \boldsymbol{I}
$$

The modified deformed gradient tensor $\overline{\boldsymbol{F}}$ is proposed by Froly ${ }^{(11)}$

$$
\overline{\boldsymbol{F}}=J^{-\frac{1}{3}} F ; \quad J:=\operatorname{det}(\boldsymbol{F}),
$$

where $J$ gives the local volume change and the notation - gives modified deformation tensor preserving volume change. Figure 1 shows the relation between the initial configuration $C$ and an intermediate configuration $\bar{C}$ defined by the volumetric deformation. The initial configuration $C$ is moved to the intermediate configuration by $J^{\frac{1}{3}} \boldsymbol{I}$. The intermediate configuration $\bar{C}$ moves to the current configuration $C$ by the modified deformation gradient tensor $\bar{F}$. Accordingly, the intermediate configuration defined by $\bar{C}$ arises from a shape-preserving volume change. Similarly, associated with the intermediate configuration, the modified right Cauchy and left Cauchy deformation tensor are defined as:

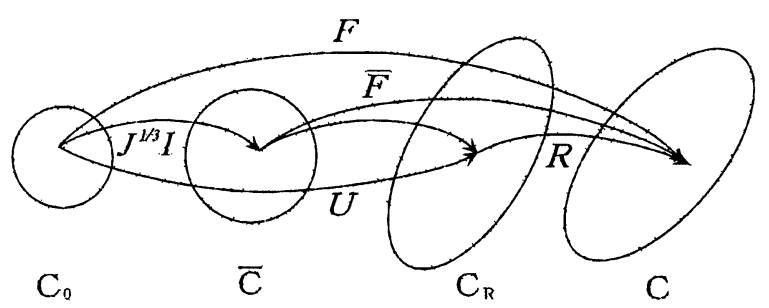

Fig. 1 Initial, current and intermediate configurations

$$
\overline{\boldsymbol{C}}=\overline{\boldsymbol{F}}^{t} \cdot \overline{\boldsymbol{F}}, \quad \overline{\boldsymbol{B}}=\overline{\boldsymbol{F}} \cdot \overline{\boldsymbol{F}}^{t}
$$

The third invariants of the above tensors, $\bar{I}_{3}$, are unity by definition.

The modified velocity gradient with respect to the intermediate configuration is obtained as:

$$
\overline{\boldsymbol{L}}=\dot{\overline{\boldsymbol{F}}} \cdot \overline{\boldsymbol{F}}^{-1}, \quad(\overline{\boldsymbol{L}})_{s}=\overline{\boldsymbol{D}}=\boldsymbol{D}^{\prime},
$$

where the notation $\bullet^{\prime}$ gives the deviatoric part of the tensor. The modified spatial rate of deformation tensor $\overline{\boldsymbol{D}}$ is the symmetric part of $\overline{\boldsymbol{L}} . \overline{\boldsymbol{D}}$ corresponds to the deviatoric part of the spatial rate of the deformation tensor $\overline{\boldsymbol{D}}^{\prime}$ and is free from volumetric deformations.

\subsection{Stress and stress rate of hyperelasticity}

A stored-energy function of hyperelasticity is defined as:

$$
W=W(\boldsymbol{C})
$$

For isotropic hyperelasticity, the stored-energy function is a function of the principal invariants of $\boldsymbol{C}$

$$
W(\boldsymbol{C})=W\left(I_{1}, I_{2}, I_{3}\right)
$$

which are calculated according to

$$
I_{1}=\boldsymbol{C}: \boldsymbol{I}, \quad I_{2}=\frac{1}{2}\left(I_{1}^{2}-\boldsymbol{C}^{2}: I\right), \quad I_{3}=\operatorname{det}(\boldsymbol{C}) .
$$

It is assumed that the stored-energy function can be decomposed additively into a volume-preserving and a purely volume-dependent term:

$$
W(\boldsymbol{C})=\bar{W}(\overline{\boldsymbol{C}})+U^{\circ}(J)
$$

The volume-preserving stored-energy function $\bar{W}$ is a function of the first and the second principal invariants of the modified right Cauchy-Green deformation tensor $\overline{\boldsymbol{C}}$ :

$$
\bar{W}(\overline{\boldsymbol{C}})=\bar{W}\left(\bar{I}_{1}, \bar{I}_{2}\right)
$$

where the principal invariants of $\overline{\boldsymbol{C}}$ are calculated according to

$$
\bar{I}_{1}=\overline{\boldsymbol{C}}: \boldsymbol{I}, \quad \bar{I}_{2}=\frac{1}{2}\left(\bar{I}_{1}^{2}-\overline{\boldsymbol{C}}^{2}: \boldsymbol{I}\right), \quad \bar{I}_{3}=1.0 .
$$

The derivative of the stored-energy function with respect to the right Cauchy-Green deformation tensor $\boldsymbol{C}$ gives the Second Piola-Kirchhoff stress tensor $\boldsymbol{S}^{0}$

$$
\boldsymbol{S}^{0}=2 \frac{\partial W(\boldsymbol{C})}{\partial \boldsymbol{C}}
$$

and the Cauchy stress $\boldsymbol{T}$ is related to it as follows:

$$
\boldsymbol{T}=\frac{1}{J} \boldsymbol{F} \cdot \boldsymbol{S}^{0} \cdot \boldsymbol{F}^{t} .
$$

The Cauchy stress $\boldsymbol{T}$ and 2nd Piola-Kirchhoff stress are decomposed into deviatoric part and mean part in the current configuration as:

$$
\boldsymbol{T}=\boldsymbol{T}^{\prime}+T_{m} \boldsymbol{I}, \quad \boldsymbol{S}^{0}=\boldsymbol{S}^{\prime 0}+\boldsymbol{S}_{m}^{0}
$$

$\boldsymbol{T}^{\prime}$ and $T_{m}$ are deviatoric and mean parts of the Cauchystress defined as:

$$
T_{m} \cdot J \cdot C^{-1}=\frac{\partial U^{\circ}(J)}{\partial C}
$$




$$
\boldsymbol{T}^{\prime}=2 \boldsymbol{F} \frac{\partial \bar{W}(\overline{\boldsymbol{C}})}{\partial \boldsymbol{C}} \boldsymbol{F}^{t}
$$

$S^{\prime 0}$ and $S_{m}^{0}$ are deviatoric and mean parts of the 2nd PiolaKirchhoff stress given by

$$
\begin{aligned}
& \boldsymbol{S}^{0}=2 \frac{\partial \bar{W}(\overline{\boldsymbol{C}})}{\partial \boldsymbol{C}}=2 \frac{\partial \bar{W}(\overline{\boldsymbol{C}})}{\partial \overline{\boldsymbol{C}}} \frac{\partial \overline{\boldsymbol{C}}}{\partial \boldsymbol{C}}, \\
& \boldsymbol{S}_{m}^{0}=\frac{\partial U^{\circ}(J)}{\partial \boldsymbol{C}} .
\end{aligned}
$$

The deviatoric part of the 2nd Piola-Kirchhoff stress is calculated as:

$$
\begin{aligned}
\boldsymbol{S}^{\prime 0} & =2\left\{\frac{\partial \bar{W}\left(\bar{I}_{1}, \bar{I}_{2}\right)}{\partial \bar{I}_{1}} J^{-\frac{2}{3}}\left(\boldsymbol{I}-\frac{1}{3} I_{1} \boldsymbol{C}^{-1}\right)\right. \\
& \left.+\frac{\partial \bar{W}\left(\bar{I}_{1}, \bar{I}_{2}\right)}{\partial \bar{I}_{2}} J^{-\frac{4}{3}}\left(I_{1} \boldsymbol{I}-\boldsymbol{C}-\frac{2}{3} I_{2} \boldsymbol{C}^{-1}\right)\right\} .
\end{aligned}
$$

The deviatoric part of the Cauchy stress in the current configuration is also calculated as:

$$
\begin{aligned}
\boldsymbol{T}^{\prime} & =\frac{2}{J}\left\{\frac{\partial \bar{W}\left(\bar{I}_{1}, \bar{I}_{2}\right)}{\partial \bar{I}_{1}} J^{-\frac{2}{3}}\left(\boldsymbol{B}-\frac{1}{3} I_{1} \boldsymbol{I}\right)\right. \\
& \left.+\frac{\partial \bar{W}\left(\bar{I}_{1}, \bar{I}_{2}\right)}{\partial \bar{I}_{2}} J^{-\frac{4}{3}}\left(I_{1} \boldsymbol{B}-\boldsymbol{B} \cdot \boldsymbol{B}-\frac{2}{3} I_{2} \boldsymbol{I}\right)\right\} .
\end{aligned}
$$

These stress have following relationship

$$
\boldsymbol{S}^{\prime 0}: C=0, \quad \boldsymbol{T}^{\prime}: \boldsymbol{I}=0 .
$$

The stress rate of the 2nd Piola-Kirchhoff stress defined by Eq. (21) is calculated using time differentiation of the right Cauchy-Green deformation tensor $\dot{\boldsymbol{C}}$ according to

$$
\dot{\boldsymbol{S}}^{\prime 0}=2\left\{\frac{\partial^{2} \bar{W}}{\partial \boldsymbol{C} \partial \boldsymbol{C}}\right\}: \dot{\boldsymbol{C}}
$$

Deviatoric part of the second Piola-Kirchhoff stress tensor is translated to the stress rate $\dot{\bar{S}}^{\prime}$ in the intermediate configuration $\bar{C}$ using $J^{1 / 3} \boldsymbol{I}$

$$
\dot{\overline{\boldsymbol{S}}}^{\prime}=J^{2 / 3} \boldsymbol{I} \cdot \dot{\boldsymbol{S}}^{\prime} \cdot \boldsymbol{I} .
$$

The Truesdell stress rate has following relationship

$$
\begin{aligned}
\stackrel{\circ}{T}_{T} & =\frac{1}{J} \boldsymbol{F} \cdot \dot{\boldsymbol{S}}^{0} \cdot \boldsymbol{F}^{t} \\
& =\dot{\boldsymbol{T}}-\boldsymbol{L} \cdot \boldsymbol{T}-\boldsymbol{T} \cdot \boldsymbol{L}^{t}+(\operatorname{tr} \boldsymbol{D}) \boldsymbol{T} .
\end{aligned}
$$

The deviatoric part of the Truesdell stress rate in the intermediate configuration is calculated as:

$$
\begin{aligned}
\stackrel{\circ}{\overline{\boldsymbol{T}}}_{T}^{\prime} & =\frac{1}{J} \overline{\boldsymbol{F}} \cdot \dot{\overline{\boldsymbol{S}}}^{\prime} \cdot \overline{\boldsymbol{F}}^{t} \\
& =\dot{\boldsymbol{T}}^{\prime}-\overline{\boldsymbol{L}} \cdot \boldsymbol{T}^{\prime}-\boldsymbol{T}^{\prime} \cdot \overline{\boldsymbol{L}}^{t}+(\operatorname{tr} \boldsymbol{D}) \boldsymbol{T}^{\prime} .
\end{aligned}
$$

The 2nd Piola-Kirchhoff stress rate is calculated as:

$$
\begin{aligned}
\dot{\overline{\boldsymbol{S}}}^{\prime} & =2\left\{\frac{\partial^{2} \bar{W}}{\partial \bar{I}_{1} \partial \bar{I}_{1}}\left(\boldsymbol{I}-\frac{1}{3} \bar{I}_{1} \boldsymbol{C}^{-1}\right) \otimes\left(\boldsymbol{I}-\frac{1}{3} \bar{I}_{1} \overline{\boldsymbol{C}}^{-1}\right)\right. \\
& +\frac{\partial^{2} \bar{W}}{\partial \bar{I}_{1} \partial \bar{I}_{2}}\left(\boldsymbol{I}-\frac{1}{3} \bar{I}_{1} \overline{\boldsymbol{C}}^{-1}\right) \otimes\left(\bar{I}_{1} \boldsymbol{I}-\overline{\boldsymbol{C}}-\frac{2}{3} \bar{I}_{2} \overline{\boldsymbol{C}}^{-1}\right) \\
& +\frac{\partial^{2} \bar{W}}{\partial \bar{I}_{2} \partial \bar{I}_{1}}\left(\bar{I}_{1} \boldsymbol{I}-\overline{\boldsymbol{C}}-\frac{2}{3} \bar{I}_{2} \overline{\boldsymbol{C}}^{-1}\right) \otimes\left(\boldsymbol{I}-\frac{1}{3} \bar{I}_{1} \boldsymbol{C}^{-1}\right) \\
& +\frac{\partial^{2} \bar{W}}{\partial \bar{I}_{2} \partial \bar{I}_{2}}\left(\bar{I}_{1} \boldsymbol{I}-\overline{\boldsymbol{C}}-\frac{2}{3} \bar{I}_{2} \overline{\boldsymbol{C}}^{-1}\right) \otimes\left(\bar{I}_{1} \boldsymbol{I}-\overline{\boldsymbol{C}}-\frac{2}{3} \bar{I}_{2} \overline{\boldsymbol{C}}^{-1}\right)
\end{aligned}
$$

$$
\begin{aligned}
& +\frac{\partial \bar{W}}{\partial \bar{I}_{1}} \frac{1}{3}\left(\frac{1}{3} I_{1} \overline{\boldsymbol{C}}^{-1} \otimes \overline{\boldsymbol{C}}^{-1}-\boldsymbol{I} \otimes \overline{\boldsymbol{C}}-\overline{\boldsymbol{C}}^{-1} \otimes \boldsymbol{I}-\bar{I}_{1} \frac{\partial\left(\overline{\boldsymbol{C}}^{-1}\right)}{\partial C}\right) \\
& +\frac{\partial \bar{W}}{\partial \bar{I}_{2}}\left(\boldsymbol{I} \otimes \boldsymbol{I}-\frac{\partial \overline{\boldsymbol{C}}}{\partial \overline{\boldsymbol{C}}}-\frac{2}{3} \bar{I}_{1} \overline{\boldsymbol{C}}^{-1} \otimes \boldsymbol{I}-\frac{2}{3} \bar{I}_{1} \boldsymbol{I} \otimes \boldsymbol{C}^{-1}\right. \\
& +\frac{2}{3} \overline{\boldsymbol{C}}^{-1} \otimes \overline{\boldsymbol{C}}+\frac{2}{3} \overline{\boldsymbol{C}} \otimes \overline{\boldsymbol{C}}^{-1}+\frac{4}{9} \bar{I}_{2} \overline{\boldsymbol{C}}^{-1} \otimes \overline{\boldsymbol{C}}^{-1} \\
& \left.\left.-\frac{2}{3} \bar{I}_{2} \frac{\partial\left(\overline{\boldsymbol{C}}^{-1}\right)}{\partial \overline{\boldsymbol{C}}}\right)\right\}: \dot{\boldsymbol{C}}=\stackrel{(}{(t)}_{i j k l}: \dot{\boldsymbol{C}}_{k l}
\end{aligned}
$$

where $\stackrel{(t)}{C}_{i j k l}$ is the consistent stiffness matrix for the total Lagrangian method. From Eqs. (24) and (27), the Truesdell stress rate in the intermediate configuration is calculated using the relation $\dot{\overline{\boldsymbol{C}}}=2 \overline{\boldsymbol{F}} \cdot \boldsymbol{D}^{\prime} \cdot \overline{\boldsymbol{F}}^{t}$

$$
\begin{aligned}
& \left(\stackrel{\circ}{\overline{\boldsymbol{T}}}_{T}^{\prime}\right)_{i j}=\frac{4}{J}\left\{\frac{\partial^{2} \bar{W}}{\partial \bar{I}_{1} \partial \bar{I}_{1}}\left(\bar{B}-\frac{1}{3} \bar{I}_{1} I\right)_{i j}\left(\bar{B}-\frac{1}{3} \bar{I}_{1} I\right)_{k l}\right. \\
& \quad+\frac{\partial^{2} \bar{W}}{\partial \bar{I}_{1} \partial \bar{I}_{2}}\left(\bar{B}-\frac{1}{3} \bar{I}_{1} I\right)\left(\bar{I}_{i j} \bar{B}-\bar{B} \cdot \bar{B}-\frac{2}{3} \bar{I}_{2} I\right)_{k l} \\
& \quad+\frac{\partial^{2} \bar{W}}{\partial \bar{I}_{2} \partial \bar{I}_{1}}\left(\bar{I}_{1} \bar{B}-\bar{B} \cdot \bar{B}-\frac{2}{3} \bar{I}_{2} I\right)_{i j}\left(\bar{B}-\frac{1}{3} \bar{I}_{1} I\right)_{k l} \\
& \quad+\frac{\partial^{2} \bar{W}}{\partial \bar{I}_{2} \partial \bar{I}_{2}}\left(\bar{I}_{1} \bar{B}-\bar{B} \cdot \bar{B}-\frac{2}{3} \bar{I}_{2} I\right)_{i j}\left(\bar{I}_{1} \bar{B}-\bar{B} \cdot \bar{B}-\frac{2}{3} \bar{I}_{2} I\right)_{k l} \\
& \quad+\frac{\partial \bar{W}}{\partial \bar{I}_{1}} \frac{1}{3}\left(\frac{1}{3} I_{1} I_{i j} I_{k l}-\bar{B}_{i j} I_{k l}-I_{i j} \bar{B}_{k l}+\bar{I}_{1} I_{i k} I_{l j}\right) \\
& \quad+\frac{\partial \bar{W}}{\partial \bar{I}_{2}}\left(\bar{B}_{i j} \bar{B}_{k l}-\bar{B}_{i k} \bar{B}_{l j}+\frac{2}{3}(\bar{B} \cdot \bar{B})_{i j} I_{k l}+\frac{2}{3} I_{i j}(\bar{B} \cdot \bar{B})_{k l}\right. \\
& \left.\left.\quad-\frac{2}{3} \bar{I}_{1} I_{i j} \bar{B}_{k l}-\frac{2}{3} \bar{I}_{1} \bar{B}_{i j} I_{k l}+\frac{4}{9} \bar{I}_{2} I_{i j} I_{k l}+\frac{2}{3} \bar{I}_{2} I_{i k} I_{l j}\right)\right\} D_{k l}^{\prime} \\
& =\stackrel{(u)}{C}_{i j k l} D_{k l}^{\prime},
\end{aligned}
$$

where $\stackrel{(u)}{\mathrm{C}}$ is the tangent stiffness matrix to be employed in the finite element formulation.

We note that the left Cauchy-Green tensor and principal invariants in Eq. (29) are volume-preserving. For an incompressible problem, the intermediate configuration corresponds to the initial configuration.

\subsection{Updated Lagrangian formulation}

We start from the following principle of stationary potential energy for the updated Lagrangian formulation

$$
\begin{aligned}
\Pi^{U}(\boldsymbol{v}) & =\int\left[U_{T}+\frac{1}{2} \boldsymbol{T}:(\nabla \boldsymbol{v} \cdot \boldsymbol{v} \nabla)\right] d V \\
& -\int \dot{\overline{\boldsymbol{t}}} \cdot \boldsymbol{v} d S-\int \rho \dot{\overline{\boldsymbol{g}}} \cdot \boldsymbol{v} d V,
\end{aligned}
$$

where $V$ is the volume occupied by the body in the current configuration and $d S$ is the surface bounding this volume. $\rho$ is the density of material in the current configuration, $\dot{\overline{\boldsymbol{t}}}$ is the rate of surface traction vector and $\dot{\overline{\boldsymbol{g}}}$ is the rate of body force vector. $U_{T}$ is the internal work rate related to the Truesdell stress rate $\stackrel{\circ}{\boldsymbol{T}}_{T}$ and its variation is given by

$$
\delta U_{T}=\stackrel{\circ}{\boldsymbol{T}}_{T}: \delta \boldsymbol{D} .
$$


From Eq. (30), the updated Lagrangian formulation of the principle of stationary potential energy using the Truesdell stress rate in the intermediate configuration is given as:

$$
\begin{aligned}
\Pi^{P}(\boldsymbol{v}) & =\int\left[U_{G}^{\prime}\left(\boldsymbol{D}^{\prime}\right)+U_{G m}\left(D_{m}\right)-2 D_{m}\left(\boldsymbol{T}^{\prime}: \boldsymbol{D}^{\prime}\right)\right. \\
& \left.-T_{m}\left(\boldsymbol{D}^{\prime}: \boldsymbol{D}^{\prime}\right)+\frac{3}{2} T_{m} D_{m}^{2}+\frac{1}{2} \boldsymbol{T}:(\nabla \boldsymbol{v} \cdot \boldsymbol{v} \nabla)\right] d V \\
& -\int \rho \dot{\overline{\boldsymbol{g}}} \cdot \boldsymbol{v} d V-\int \dot{\overline{\boldsymbol{t}}} \cdot \boldsymbol{v} d S
\end{aligned}
$$

where $U_{G}$ and $U_{G m}$ have following relationships

$$
\delta U_{G}^{\prime}=\stackrel{\circ}{\overline{\boldsymbol{T}}_{T}^{\prime}}: \delta \boldsymbol{D}^{\prime}, \quad \delta U_{G m}=3 \dot{T}_{m} \cdot \delta D_{m} .
$$

When a linear volumetric stress-strain relationships is assumed, $U_{G m}$ is calculated as $U_{G m}=9 \kappa_{0} J D_{m}^{2}$, where $\kappa_{0}$ is the balk modulus ${ }^{(12)}$. The virtual work expression for the mixed method is obtained using Lagrangian multipliers as:

$$
\begin{aligned}
\int & {\left[\left\{\frac{\partial U_{G}^{\prime}}{\partial \boldsymbol{D}^{\prime}}-2(\boldsymbol{v} \nabla)_{m} \boldsymbol{T}^{\prime}-2 T_{m} \boldsymbol{D}^{\prime}\right\}: \delta \boldsymbol{D}^{\prime}\right.} \\
& +3\left\{\dot{T}_{m}+T_{m}(\boldsymbol{v} \nabla)_{m}-\frac{2}{3}\left(\boldsymbol{T}^{\prime}: \boldsymbol{D}^{\prime}\right)\right\} \delta(\boldsymbol{v} \nabla)_{m} \\
& \left.+\boldsymbol{T}:(\nabla \boldsymbol{v} \cdot \delta \boldsymbol{v} \nabla)-3\left\{\frac{1}{3 J_{\kappa_{0}}} \dot{T}_{m}-(\boldsymbol{v} \nabla)_{m}\right\} \delta \dot{T}_{m}\right] d V \\
& =\int \rho \dot{\overline{\boldsymbol{g}}} \cdot \delta \boldsymbol{v} d V+\int \dot{\overline{\boldsymbol{t}}} \cdot \delta \boldsymbol{v} d S_{t}
\end{aligned}
$$

where $\dot{T}_{m}$ is the time derivative of mean part of the Cauchy stress and it correspond to $(\dot{\boldsymbol{T}}: \boldsymbol{I}) / 3$.

To apply selective integration, the stress and strain in Eq. (32) are decomposed into the mean and deviatoric parts and different integration rules are applied according to:

$$
\begin{aligned}
\int_{F . I .} & {\left[{\stackrel{\circ}{\overline{\boldsymbol{T}}^{\prime}}}_{T}: \delta \boldsymbol{D}^{\prime}-2 \delta D_{m}\left(\boldsymbol{T}^{\prime}: \boldsymbol{D}^{\prime}\right)\right.} \\
& \left.-2 D_{m}\left(\boldsymbol{T}^{\prime}: \delta \boldsymbol{D}^{\prime}\right)+\boldsymbol{T}^{\prime}:(\nabla \delta \boldsymbol{v} \cdot \boldsymbol{v} \nabla)\right] d V \\
& +\int_{R . I .}\left[9 \kappa_{0} D_{m} \delta D_{m}-2 T_{m}\left(\boldsymbol{D}^{\prime}: \delta \boldsymbol{D}^{\prime}\right)\right. \\
& \left.+3 T_{m} D_{m} \delta D_{m}+T_{m} \operatorname{tr}(\nabla \delta \boldsymbol{v} \cdot \boldsymbol{v} \nabla)\right] d V \\
& =\int p \dot{\overline{\boldsymbol{g}}} \cdot \delta \boldsymbol{v} d V+\int \dot{\overline{\boldsymbol{t}}} \cdot \delta \boldsymbol{v} d S,
\end{aligned}
$$

where the notation F.I. and R.I. are the full and reduced integration rules, respectively.

Here, the velocity vector $\boldsymbol{v}$, the rate of deformation tensor $\boldsymbol{D}$ and pressure stress rate $\dot{T}_{m}$ are expressed using node velocity $\stackrel{(e)}{\dot{\psi}}$ and node pressure $\stackrel{(e)}{\dot{T}}_{m}$ discretely as:

$$
\begin{aligned}
& v_{i}=\stackrel{(u)}{N}_{i}^{(m)} \stackrel{(e)}{\dot{\psi}}_{(m)},(\nabla v)_{i j}=A_{i j}^{(m)} \stackrel{(e)}{\dot{\psi}_{(m)},}, D_{i j}^{\prime}=H_{i j}^{\prime(m)} \stackrel{(e)}{\dot{\psi}}_{(m)}, \\
& D_{m}=H_{m}^{(m)} \cdot \stackrel{(e)}{\psi}_{(m)}, \dot{T}_{m}=\stackrel{(u)}{M}^{(q)} \cdot{\stackrel{(e)}{T_{m}}}_{(q)},
\end{aligned}
$$

where $\stackrel{(u)}{N}_{i}^{(m)}, A_{i j}^{(m)}$ and ${H^{\prime}}_{i j}^{(m)}$ are the shape functions for velocity and deformation. $\stackrel{(u)}{M}^{(q)}$ is the shape function for pressure stress. $m$ and $q$ are number of node in an element. The stiffness matrix of the mixed method is then given by substituting the discrete deformation and stress tensors in Eq. (34):

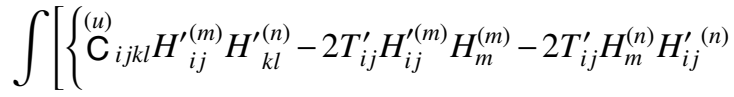

$$
\begin{aligned}
& \left.-2 T_{m} H_{i j}^{\prime(m)} H_{i j}^{\prime(n)}+3 T_{m} H_{m}^{(n)} H_{m}^{(m)}+T_{i j} A_{i h}^{(n)} A_{j h}^{(m)}\right\} \dot{\psi}_{(n)} \\
& \left.+3 H_{m}^{(m)} \stackrel{(u)}{M}^{(r)} \stackrel{(e)}{T}_{m(r)}\right] d V_{e} \\
& =\int \rho \dot{\bar{g}}_{i} \cdot \stackrel{(u)}{N}_{i}^{(m)} d V_{e}+\int \dot{\bar{t}}_{i} \cdot \stackrel{(u)}{N}_{i}^{(m)} d S_{e}, \\
& \int\left[3 H_{m}^{(n)} \stackrel{(u)}{M}^{(q)} \stackrel{(e)}{\dot{\psi}}_{(n)}-\frac{1}{J K_{0}} \stackrel{(u)}{M}^{(q)} \stackrel{(u)}{M}^{(r)} \stackrel{(e)}{T}_{m(r)}\right] d V_{e}=0,
\end{aligned}
$$

where $n$ and $r$ are also number of the velocity node and the pressure stress. $V_{e}$ and $S_{e}$ are volume and surface of the element, respectively.

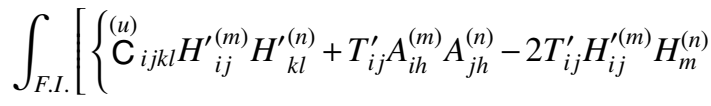

$$
\begin{aligned}
& \left.\left.-2 T_{i j}^{\prime} H_{m}^{(n)} H_{i j}^{\prime(n)}\right\}^{(e)} \dot{\psi}_{(n)}\right] d V_{e} \\
& +\int_{\text {R.I. }}\left[\left\{9 \kappa_{0} J H_{m}^{(m)} H_{m}^{(n)}-2 T_{m} H_{i j}^{(m)} H_{i j}^{(n)}\right.\right. \\
& \left.+3 T_{m} H_{m}^{(m)} H_{m}^{(n)}+T_{m} A_{i j}^{(m)} A_{i j}^{(n)} \stackrel{(e)}{\psi}_{(n)}\right] d V_{e} \\
& =\int \rho \dot{\bar{g}_{i}} \cdot \stackrel{(u)}{N}_{i}^{(m)} d V_{e}+\int \dot{\bar{t}}_{i} \cdot{ }^{(u)} N_{i}^{(m)} d S_{e}
\end{aligned}
$$

\subsection{Total Lagrangian formulation}

The principle of stationary potential energy for the total Lagrangian formulation is given by:

$$
\Pi^{T}(\boldsymbol{u})=\int W d V_{0}-\int \boldsymbol{t} \cdot \boldsymbol{u} d S_{0}-\int \rho_{0} \boldsymbol{g} \cdot \boldsymbol{u} d V_{0},
$$

where $V_{0}$ is the volume occupied by the body in the initial configuration and $S_{0}$ is the surface in the initial configuration. $\boldsymbol{t}$ and $\boldsymbol{g}$ are the traction and body force vector, respectively, and $\rho_{0}$ is the density of material in initial configuration. For a Total Lagrangian formulation, a mixed displacement-pressure method reads:

$$
\begin{aligned}
& \int\left[\left\{\frac{\partial \bar{W}}{\partial \boldsymbol{C}}+p \frac{\partial J}{\partial \boldsymbol{C}}\right\}: \delta \boldsymbol{C}+\left\{J-1-\frac{\partial U_{C}^{\circ}(p)}{\partial p}\right\} \delta p\right] d V \\
& =\int \boldsymbol{t} \cdot \delta \boldsymbol{u} d S+\int \rho_{0} \boldsymbol{g} \cdot \delta \boldsymbol{u} d V
\end{aligned}
$$

where the Lagrangian multiplier $p$ corresponds to the pressure stress and is related to the updated formulation via $p=T_{m}$.

$$
\begin{aligned}
& \int\left[\left({ } ^ { ( t ) } { } _ { i j k l } \{ B _ { i j } ^ { l ( m ) } + 2 B _ { i j } ^ { n l ( m o ) } \stackrel { ( e ) } { u _ { ( o ) } } \} \left\{\left\{B_{k l}^{l(n)}+2 B_{k l}^{n l(n p)} \stackrel{(e)}{u}_{(p)}\right\}\right.\right.\right. \\
& \left.\quad+S_{i j} B_{i j}^{n l(m n)}\right) \Delta \stackrel{(e)}{u}_{(n)}
\end{aligned}
$$




$$
\begin{aligned}
& \left.+\frac{J}{2}\left(C^{-1}\right)_{i j}\left\{B_{i j}^{l(m)}+2 B_{i j}^{n l(m n)} \stackrel{(e)}{u}_{(n)}\right\} \stackrel{(t)}{M}^{(r)} \Delta \stackrel{(e)}{p}_{(r)}\right] d V_{e} \\
& =\int\left(t_{i}+\Delta t_{i}\right) \stackrel{(t)}{N_{i}}{ }^{(m)} d S_{e}+\int \rho_{0}\left(g_{i}+\Delta g_{i}\right) \cdot \stackrel{(t)}{N}_{i}^{(m)} d V_{e} \\
& -\int\left[\frac{1}{2} S_{i j}^{(m)}\left\{B_{i j}^{l(m)}+2 B^{n l(m n)^{(e)}}{ }_{(n)}\right\}\right] d V_{e} \\
& \int\left\{\frac{J}{2}\left(C^{-1}\right)_{i j}\left\{B_{i j}^{l(m)}+2 B_{i j}^{n l(m n)} \stackrel{(e)}{u}{ }_{(n)}\right\} \Delta \Delta^{(e)}{ }_{(m)} \stackrel{(t)}{M}{ }^{(r)}\right. \\
& \left.-\left(\frac{1}{\kappa_{0}} \stackrel{(t)}{M}^{(q)} \stackrel{(t)}{M}^{(r)} \Delta \stackrel{(e)}{P}_{(q)}\right)\right\} d V_{e} \\
& =-\int\left[\left\{(J-1)-\frac{p}{\kappa_{0}}\right\}^{(t)}{ }^{(r)}\right] d V_{e} \\
& u_{i}=\stackrel{(t)}{N}_{i}^{(m)} \cdot \stackrel{(e)}{u}_{(m)}, \quad p=\stackrel{(t)}{M}^{(q)} \cdot \stackrel{(e)}{p}_{(q),}, \\
& C_{i j}=\left(B^{l(m)}+B^{n l(m n)} \stackrel{(e)}{u}_{(n)}\right) \cdot \stackrel{(e)}{u}_{(m)}+\delta_{i j},
\end{aligned}
$$

The commercial FEM code ABAQUS is based on the Updated Lagrangian formulation using the virtual work principle $^{(13)}$

$$
\int \boldsymbol{T}: \delta \boldsymbol{D} d V-\int \overline{\boldsymbol{t}} \cdot \delta \boldsymbol{v} d S-\int \rho \boldsymbol{g} \cdot \delta \boldsymbol{v} d V .
$$

\section{Numerical Analysis}

The objective of this section is to assess the formulation and numerical implementation of the formulations presented in the preceding sections.

\subsection{Time stepping algorithm}

The Newton-Raphson and the forward-Euler algorithm were applied for the time stepping algorithm of the updated Lagrangian formulation. For the total Lagrangian formulation, only the Newton-Raphson algorithm was applied. In the Newton-Raphson algorithm, the iteration calculation would be repeated until the un-constrained node force would be small.

In the forward-Euler algorithm, $\boldsymbol{u}_{n}$ and $\boldsymbol{u}_{n+1}$ are defined as displacement in $n$ and $n+1$ steps, where $\boldsymbol{u}_{n}$ is already calculated and $\boldsymbol{u}_{n+1}$ is unknown. Then, $\boldsymbol{u}_{n+1}$ is updated according to

$$
\boldsymbol{u}_{n+1}=\boldsymbol{u}_{n}+\Delta t \cdot \boldsymbol{v} .
$$

The deformation gradient tensor $\boldsymbol{F}$ and the Cauchy-stress are updated according to

$$
\begin{aligned}
\boldsymbol{F}_{n+1} & =\boldsymbol{F}_{n}+\Delta t \cdot \boldsymbol{F}_{n} \cdot \frac{\partial \boldsymbol{v}}{\partial \boldsymbol{x}_{n}}, \\
\boldsymbol{T}_{n+1} & =\boldsymbol{T}_{n} \\
& +\Delta t \cdot\left\{\stackrel{\circ}{T}_{T}+\boldsymbol{L} \cdot \boldsymbol{T}_{n}+\boldsymbol{T}_{n} \cdot \boldsymbol{L}^{t}-(\operatorname{tr} \boldsymbol{D}) \boldsymbol{T}_{n}\right\} .
\end{aligned}
$$

Node force is given by integration of Eq. (30) for an element.

$$
\stackrel{(e)}{f}_{n+1}=\int \boldsymbol{T}_{n+1}: \boldsymbol{H} d V_{e}
$$

\subsection{Numerical example}

3.2. Plane strain deformation of square block Numerical calculations of plane strain tensile deformation of square block were conducted to assess proposed formulation. The square block is $20 \mathrm{~mm}$ of width and $20 \mathrm{~mm}$ height. One plane-strain 4-node element is used for modeling. Following Mooney-Rivlin model is introduced for material model ${ }^{(14),(15)}$

$$
\bar{W}(\overline{\boldsymbol{C}})=C_{1}\left(\bar{I}_{1}-3.0\right)+C_{2}\left(\bar{I}_{2}-3.0\right)
$$

Material parameters are assumed as:

$$
C_{1}=1.0 \mathrm{MPa}, \quad C_{2}=0.5 \mathrm{MPa}
$$

Volumetric stress-strain relation assumed incompressible. Then the selective integration method is not able to apply, because it can be applied only for compressible condition.

Both of the forward-Euler and Newton-Raphson algorithms were applied for the updated Lagrangian formulation. For 1 incremental calculation of the NewtonRaphson algorithm, $50 \%$ of tensile strain $\Delta \varepsilon_{v}$ in vertical direction and 2 times iteration calculations were applied. $1 \%$ of tensile strain $\left(\Delta \varepsilon_{v}\right)$ and 2 times iteration calculations were applied for forward-Euler algorithm. FEM mesh, stress-strain relation and volume-deformation relation are shown in Fig. 2.

Stress-strain relations computed by the NewtonRaphson algorithm are correspond to theoretical results. The total and updated Lagrangian formulation shows the same results, if the Newton-Raphson algorithm was applied. The updated Lagrangian formulation applied the forward-Euler algorithm show higher tensile load and smaller volume compared with the Newton-Raphson algorithm.

It would be hard for the forward-Euler algorithm to keep incompressible condition completely even if the incremental deformation would be very small.

3.2.2 Deformation of a rectangle block Deformation of a simple rectangle block was considered. The rectangle block has $200 \mathrm{~mm}$ of width and depth and $400 \mathrm{~mm}$ of height. Linear volumetric stress-strain relationships is considered and the bulk modulus $\kappa_{0}$ is assumed as $1000 \mathrm{MPa}$. 8-node solid element was applied and the boundary conditions include constrained top and bottom surface.

For time stepping algorithm, the Newton-Raphson algorithm was applied for mixed method by total and updated Lagrangian formulations and the forward-Euler algorithm was applied for selected integration method by updated Lagrangian formulation.

First, a horizontal deformation $\left(u_{h}\right)$ of $400 \mathrm{~mm}$ was applied at the top surface. For the Newton-Raphson algorithm, $8 \mathrm{~mm}$ of incremental horizontal deformation and one iteration was applied per step. For the forward-Euler algorithm, $4 \mathrm{~mm}$ of deformation was applied for one step. Plots of the resulting load-displacement curves are shown 


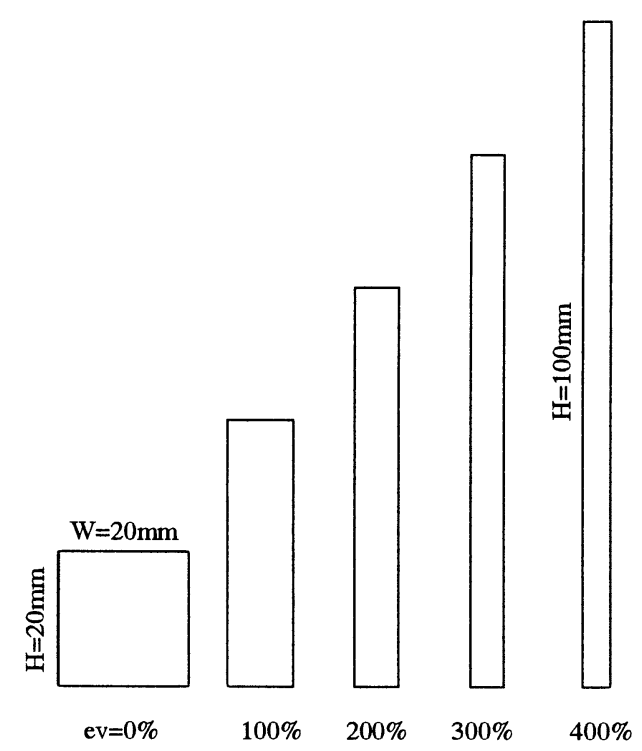

(a) Tensile deformation of one element

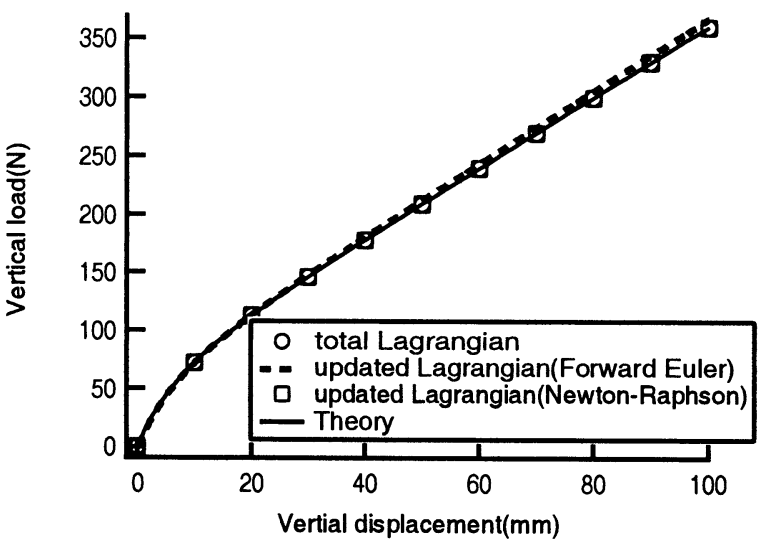

(b) Relation between vertical load and displacement

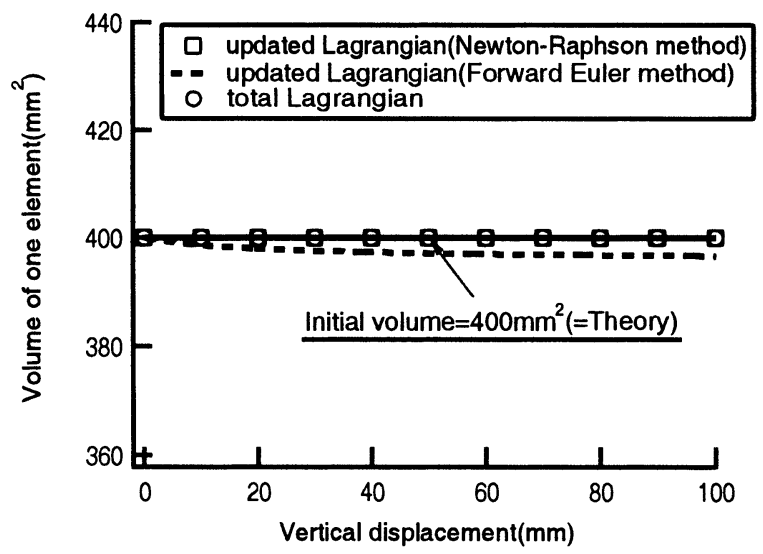

(c) Relation between area and vertical displacement

Fig. 2 Relationships above vertical load, area of one element and vertical displacement in plane strain and incompressible conditions $\left(C_{1}=1.0, C_{2}=0.5[\mathrm{MPa}], \kappa_{0}=\infty\right)$

in Fig. 3, where the horizontal strain has been calculated as $e_{h}=u_{h} / 200.0$. All curves looks similar. However, a numerical error was observed in the results of forward Euler method, because it was calculated without iterations.

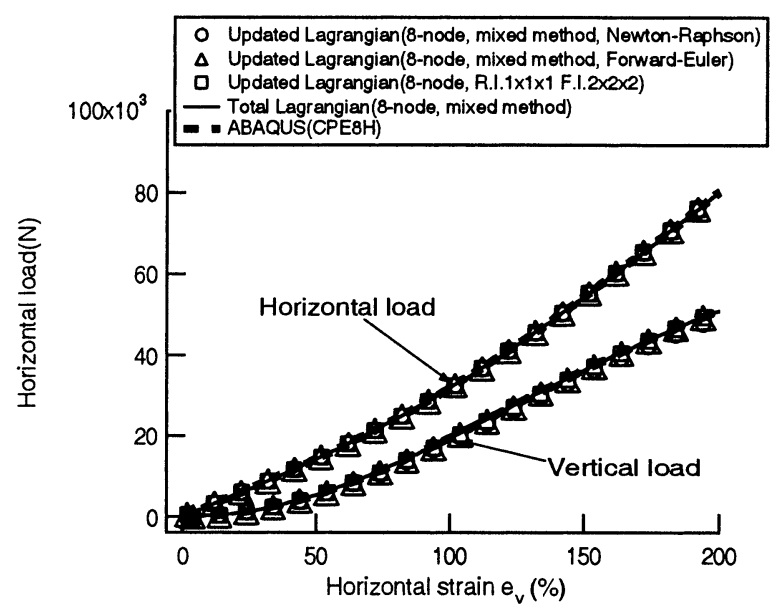

Fig. 3 Relationships between vertical load and vertical strain of 3-dimensional analysis $\left(\kappa_{0}=1000 \mathrm{MPa}\right)$

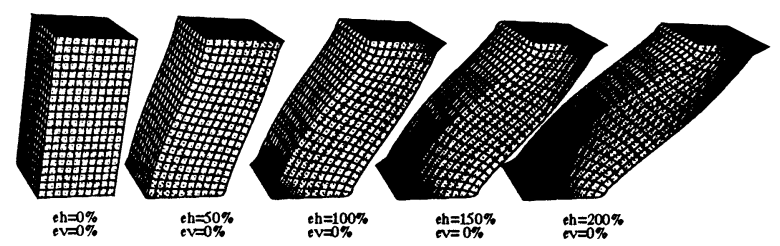

(a) Updated Lagrangian mixed formulation (Newton-Raphson)

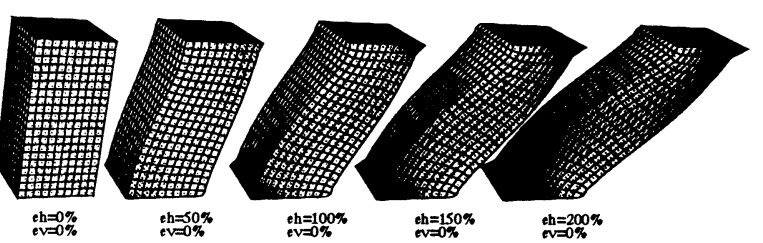

(b) Updated Lagrangian formulation and selective integration method (Forward-Euler)

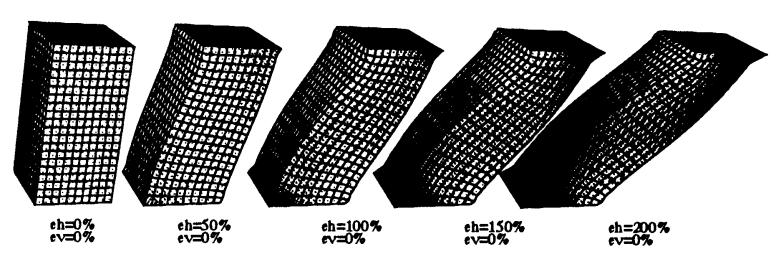

(c) Total Lagrangian formulation (Newton-Raphson)
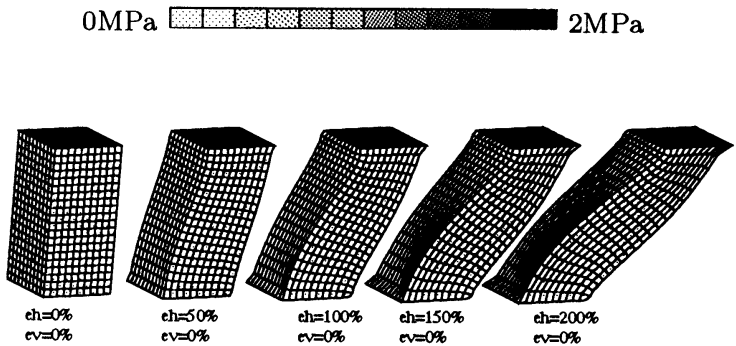

(d) ABAQUS

Fig. 4 Shear deformation of rectangle block

Finite element meshes with a mean stress contour corresponding to $100 \mathrm{~mm}, 200 \mathrm{~mm}, 300 \mathrm{~mm}, 400 \mathrm{~mm}$ of horizontal displacement are shown in Fig. 4. Neither lock- 


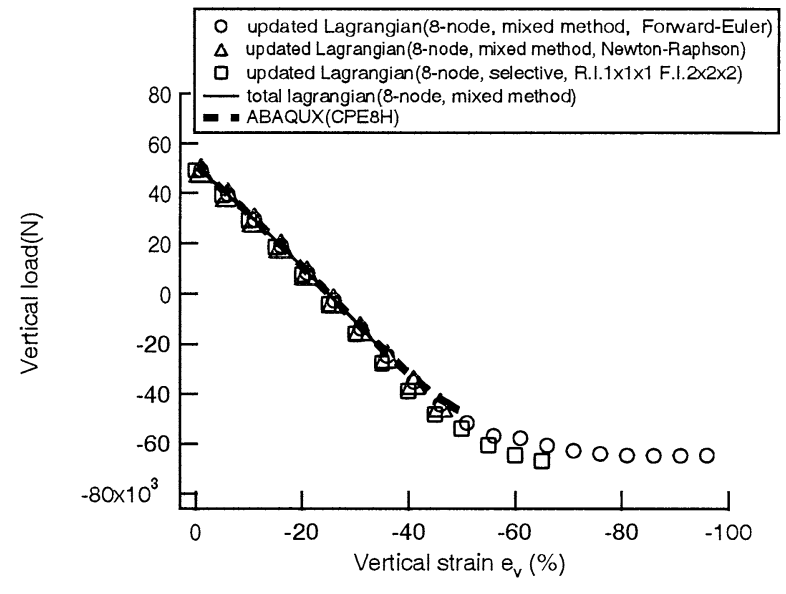

Fig. 5 Relationships between vertical load and vertical strain of 3-dimensional analysis $\left(\kappa_{0}=1000 \mathrm{MPa}\right)$

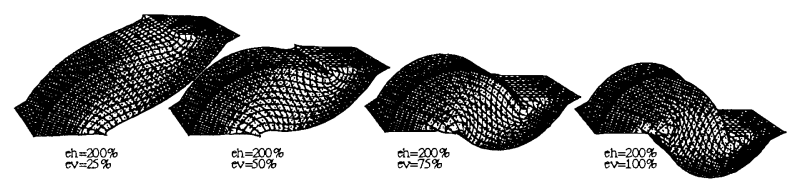

(a) Updated Lagrangian mixed formulation (Forward-Euler)

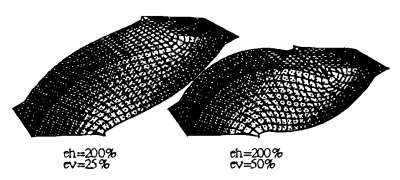

(b) Updated Lagrangian formulation and selective integration method

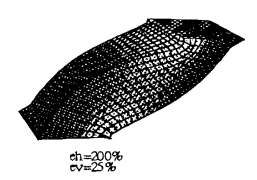

(c) Total Lagrangian mixed method

Fig. 6 Deformation of compressed rectangle block under $400 \mathrm{~mm}$ shear deformation

ing, nor hourglass modes were observed and the deformation patterns calculated from the various formulations are rather similar.

Next, a vertical deformation $\left(u_{v}\right)$ was applied after $400 \mathrm{~mm}$ of horizontal deformation until divergence of the calculation occurred. $8 \mathrm{~mm}$ of incremental vertical deformation and one iteration per step were applied in case of the Newton-Raphson algorithm and increments of $4 \mathrm{~mm}$ were applied for the forward Euler algorithm. Plots of the resulting load-deformation curves are shown in Fig. 5, where the vertical strain has been calculated as $e_{v}=u_{v} / 400.0$. FEM meshes with the mean stress contour corresponding to $100 \mathrm{~mm}, 200 \mathrm{~mm}, 300 \mathrm{~mm}, 400 \mathrm{~mm}$ of vertical displacement are shown in Fig. 6.

The load-displacement curves for the NewtonRaphson algorithm show convergence characteristics similar to those of ABAQUS. The forward-Euler algorithm
Table 1 Geometrical data of the thick natutal rubber bearing

\begin{tabular}{l|l}
\hline Diameter of rubber bearing & $252 \mathrm{~mm}$ \\
Thickness of one rubber layer & $31.5 \mathrm{~mm}$ \\
Number of rubber layers & 6 \\
Total thickness of rubber layers & $189 \mathrm{~mm}$ \\
Thickness of one steel layer & $2 \mathrm{~mm}$ \\
Number of steel layers & 5 \\
Loaded area & $50200 \mathrm{~mm}^{2}$ \\
\hline
\end{tabular}

Table 2 Coefficients of the Eq. (52)

\begin{tabular}{llllll}
\hline $\mathrm{A}$ & $C_{A 1}$ & $C_{A 2}$ & $C_{A 3}$ & $C_{A 4}$ & $C_{A 5}$ \\
\hline 1 & $2.05 \times 10^{-1}$ & $1.32 \times 10^{-2}$ & $2.35 \times 10^{-4}$ & $1.71 \times 10^{-1}$ & -2.12 \\
2 & $1.35 \times 10^{-2}$ & $-1.61 \times 10^{-3}$ & $6.32 \times 10^{-5}$ & $-6.87 \times 10^{-2}$ & -6.44 \\
\hline
\end{tabular}

shows slightly better convergence behavior, but some elements in the corner exhibit suspicious deformation patterns.

3.2.3 Simulation of isolated rubber Loading tests on a prototype rubber bearing were conducted to assess the applicability of the computer code. The bearing consists six rubber layers and five reinforcing steel plates. The diameter is $252 \mathrm{~mm}$. The thicknesses of the rubber layers and reinforcing steel platens are $31.5 \mathrm{~mm}$ and $2 \mathrm{~mm}$, respectively. These measures have been chosen such that the rubber can undergo large deformations and steel platens can experience bending, but are outside the normal design of rubber bearings used for isolation of buildings. The geometrical data are summarized in Table 1. The rubber used for the specimen is a natural rubber with a very small material damping. The potential energy function of the natural rubber material has been evaluated by coupon tests ${ }^{(8)}$ :

$$
\begin{aligned}
& \frac{\partial \bar{W}^{\circ}}{\partial \bar{I}_{A}}=C_{A 1}+C_{A 2} \cdot\left(\bar{I}_{A}-3\right)+C_{A 3} \cdot\left(\bar{I}_{A}-3\right)^{2} \\
& \quad+C_{A 4} \cdot \exp \left\{C_{A 5} \cdot\left(\bar{I}_{A}-3\right)\right\} \quad(A=1,2)
\end{aligned}
$$

Material parameters in Eq. (52) are shown in Table 2. The volumetric stress-strain relation is assumed linear and the Poisson's ratio is defined as 0.4995 . The boundary conditions include a constrained bottom surface, a vertical force of $0 \mathrm{MPa}, 1.22 \mathrm{MPa}$ and $2.45 \mathrm{MPa}$ and shear deformation applied to the top surface simultaneously. The finite element mesh is divided into 4 elements in the vertical and radial direction and into 12 elements in the circumferential direction.

Selective integration and the forward-Euler algorithm are applied because of less number of degree of freedom and the increased numerical stability.

For the constitutive equation of reinforcing steel platens, the Jaumann rate of Cauchy stress and an isotropic hardening law are used. The parameters of the hardening law were determined from a tensile loading test on a mild 

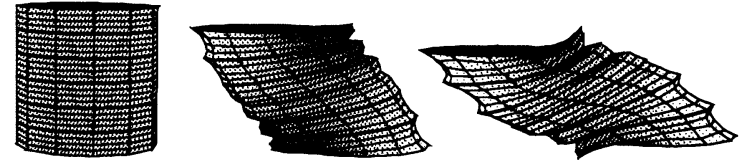

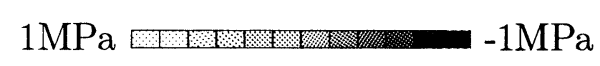

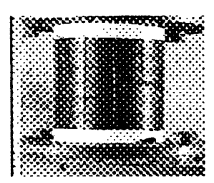

$e_{h}=0 \%$

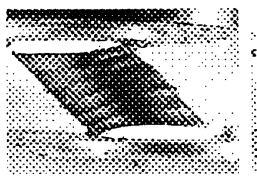

$e_{h}=130 \%$

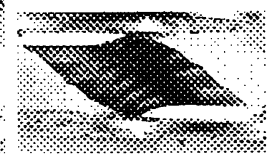

$e_{h}=160 \%$
Fig. 7 Comparison loading tests with finite element analysis $(P=1.22 \mathrm{MPa})$

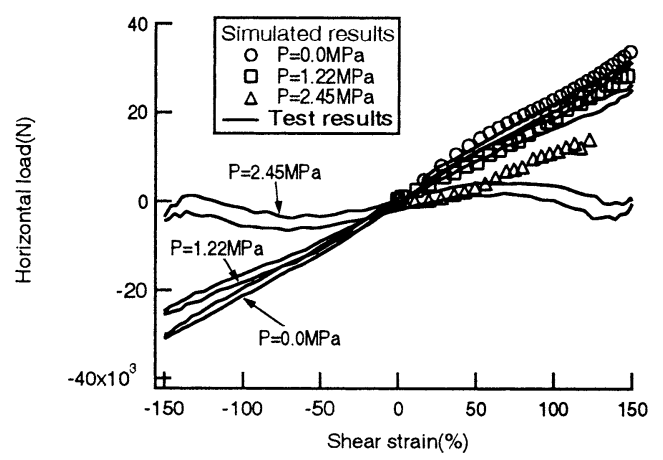

(a) Relationships between horizontal load and shear strain

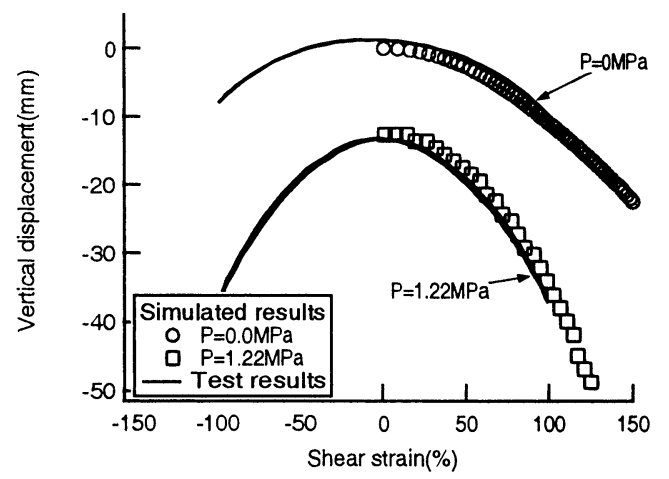

(b) Relationships between vertical displacement and horizontal displacement

Fig. 8 Comparion of simulated results with experiments

steel. The relationship between tensile stress and strain is expressed by:

$$
\sigma_{t}=179.0+345.0[1.0-\exp (-3.40 \zeta)] \quad[\mathrm{MPa}]
$$

where $\sigma_{t}$ is tensile stress and $\zeta$ is the equivalent plastic strain. For the elastic properties of the steel, the Young's modulus is taken as $200 \mathrm{GPa}$ and the Poisson's ratio is taken as 0.3 .

The pictures of loading experiment and of the finite element mesh with mean stress contours after the application of 1.25 MPa of vertical load are shown in Fig. 7. The numerical calculation well predicts the large deformation of the rubber layers and the bending behavior of the reinforcing steel platen.

Relationships between horizontal deformation and horizontal load and relationships between horizontal and vertical deformation are shown in Fig. 8. In the case of application of $2.42 \mathrm{MPa}$ vertical load, the vertical displacement could not be measured because it was beyond the capacity of displacement sensor.

In the other cases, the numerical calculation well predicts the vertical displacement due to horizontal displacement. These agreements show the applicability of the formulation to predict coupled behavior of large deformation of rubber material and elasto-plastic deformation of mild steel.

\section{Conclusions}

Finite element equations based on the Truesdell stress rate of isotropic compressible hyperelasticity are presented. By introducing an intermediate volume-preserving configuration, the stress rate and the consistent stiffness matrix were described using only the deformation in the intermediate configuration. This stress rate refers the same configuration as that of the elasto-plastic constitutive equation.

By comparing with Total Lagrangian formulation and a commercial FEM code, similar accuracy and response characteristics were observed, provided of course that the same algorithm was applied.

Finally, loading test of a prototype of rubber bearing was simulated to assess the applicability of our formulation to this class of problems. Deformation and loaddeformation responses are well predicted even if rubber and steel material exhibit large deformations.

\section{References}

( 1 ) Hibbitt, H.D., Marcal, P.V. and Rice, J.R., A Finite Element Formulation for Probrems of Large Strain and Large Displacement, International Journal of Solids Structures, Vol.6 (1970), pp.1069-1086.

( 2 ) Bathe, K.J., Ramm, E. and Wilson, E.L., Finite Element Formulations for Large Deformation Dynamic Analysis, International Journal for Numerical Methods in Engineering, Vol.9 (1975), pp.353-386.

( 3 ) Bathe, K.J., Finite Element Procedures, (1996), Prentice-Hall.

( 4 ) Hughes, T.J.R., The Finite Element Method, Linear Static and Dynamic Finite Element Analysis, (2000), Dover Publications.

( 5 ) Nagtegaal, J.C., Parks, D.M. and Rice, J.R., On Numerically Accurate Finite Element Solutions in the Fully Plastic Range, Computer Methods in Applied Mechanics and Engineering, Vol.4 (1974), pp.153-177.

( 6 ) Simo, J.C. and Hughes, T.J.R., Computational Inelasticity, (1997), Springer.

( 7 ) Simo, J.C., Taylor, R.L. and Pister, K.S., Variational 
and Projection Methods for the Volume Constant in Finite Deformation Elast-Plasticity, Computer Methods in Applied Mechanics and Engineering, Vol.51 (1985), pp.177-208.

( 8 ) Seki, W., Fukahori, Y., Iseda, Y. and Matsunaga, T., A Large-Deformation Finite -Element Analysis for Multilayer Elastomeric Bearings, Transaction of a Meeting of the Rubber Division, (1970), pp.856-870, American Chemical Society.

(9) Watanabe, H. and Hisada, T., Mixed Finite Element Analysis of Incompressible Hyperelastic Materials, Transaction of JSME, Vol.62, No.595 (1996), pp.745752.

(10) Matsuda, A., Three-Dimensional Finite Element Analysis of Laminated Rubber Bearings Using the Selective Integration Method, JSME Int. J. Ser.A, Vol.42, No.1 (1999), pp.40-48.
(11) Flory, P.J., Thermodynamic Relations for High Elastic Materials, Transactions of the Faraday Society, Vol.57 (1961), pp.829-838.

(12) Watanabe, O., Constitutive Equations and Mixed Variational Principle of Hyperelasticity Using Rotationless Strain, IUTAM Symposium Hannover/Germany, (1991), pp.227-236.

(13) Hibbitt, Karlsson \& Sorensen: ABAQUS Theory Manual Version 5.8, (1997).

(14) Rivlin, R.S., Large Elastic Deformation of Isotropic Materials IV Further Developments of the General Theory, Philosophical Transaction, Vol.A.241 (1948), pp.379-397.

(15) Rivlin, R.S. and Saunders, D.W., Large Elastic Deformation of Isotropic Materials VII Experiments on the Deformation of Rubber, Philosophical Transaction, Vol.243 (1951), pp.251-288. 